\title{
THE CHEMOTHERAPY OF NON-TUBERCULOUS DISEASES OF THE CHEST
}

\author{
By Horace Joules, M.D., F.R.C.P. \\ Central Middlesex Hospital
}

The increasing use of chemotherapy in lung infections has caused a renewal of interest in their bacteriological investigation. Clarity of thought, a deeper understanding of pathology and a vastly improved prognosis naturally result. However it is essential that our interest in this aspect of causation should not obscure the more important social and personal factors. Much acute and chronic chest disease is determined by conditions at home and work which are remediable by our society. Overwork, anxiety and malnutrition are important personal factors which should always be investigated and abolished where possible. Unless these environmental and individual contributions to ill health are eradicated treatment remains an end in itself and not a weapon in a campaign to eliminate preventable disease.

The choice of chemotherapy at present is almost embarrassing and there is a tendency to rush and use each new antibiotic as it becomes available. Therapeutic trials with controls are difficult to conduct over a sufficiently long period, because of excessive claims for each new drug in both the medical and lay press. These claims create a demand by profession and public, and tend to exaggerate the therapeutic efficiency and to neglect the toxic effects. Most physicians will have seen patients who have suffered severely from the too prolonged administration of the newer antibiotics. An example of this was a female patient, aged 60 , admitted with severe dehydration resulting from diarrhoea. This had commenced four days after giving chloramphenicol for a mild pneumonia. The drug was continued for $\mathbf{2 8}$ days in an attempt to cure the suspected intestinal infection. The patient eventually recovered after discontinuing that treatment and giving effective therapy both for the dehydration and for the gross glossitis and enterocolitis which chloramphenicol had caused.

Economy in the use of this wide range of drugs can be consistent with scientific assessment and highest recovery rates. We must be prepared to say why we are using a certain antibiotic in just the same way as the surgeon should be able to justify every incision he makes. Blunderbuss therapy can diminish the effectiveness of the agents used. This is well seen in the combined use of penicillin and aureomycin in bacterial infection. Used singly their effect in treatment of some conditions is much better than in combination, and this has been confirmed in laboratory experiment (Gunnison, Coleman and Jawitz, r950a, r95ob). These findings have been repeated by other workers. The bactericidal action of penicillin appears to be exerted on the multiplying organism and this multiplication is inhibited by the bacteriostatic action of aureomycin. Present knowledge suggests that while penicillin and streptomycin may act synergistically, showing a more than additive effect, the combination of either of these drugs with chloramphenicol, aureomycin or terramycin may have the opposite result. The cost of seven days' effective dosage in hospital of commonly used substances is:-

Tab. Sulphamerazine, 4 g. and I g. every eight hours ... . . . . .

Tab. Sulphatriad, $2 \ddot{g}$. and $\dot{I}$ g. every four hours
Sol. Penicillin, 200,000 units six-hourly Dihydrostreptomycin Sulph., 2 g. daily .. Choramphenicol, 2 g. and I g. six-hourly .. Aureomycin, 2 g. and I g. six-hourly

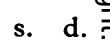

This factor should never prevent the full use of any method when indicated as a life-saving measure or when the length of illness is likely to be effectively reduced, but considerations of price must be used in conjunction with others when deciding on alternative treatments.

\section{The Treatment of Pneumonia}

For the purpose of our present discussion pneumonia can be divided into (a) bacterial and (b) virus.

(a) Bacterial pneumonia results in this country from infection by the following organisms:-

I. Pneumococci.

2. Streptococci.

3. Staphylococci.

4. B. Friedlander.

5. Haemophilus influenzae.

6. Mixed infections of the above, associated with normal inhabitants of the mouth and upper respiratory passages. 
Wherever possible treatment should be preceded by sending sputum or a laryngeal swab for bacterial investigation.

Pneumococci and streptococci are by far the commonest organisms to be dealt with when pneumonia occurs unassociated with an influenzal epidemic. These are almost always sulphonamidesensitive, consequently oral therapy will be effective. The best drug in this series, in our hands, has been sulphamerazine. Its routine use is advocated in those with lobar pneumonia whose general condition is reasonably good and who are capable of taking 6 pt. (3 to 4 l.) of fluid daily. A loading dose of $3 \mathrm{gm}$. followed by $2 \mathrm{gm}$. and subsequently I gm. eight-hourly is sufficient for the adult patient. It is rapidly absorbed from the small intestine, the toxic effects are minimal and if urinary output is maintained at $5^{\circ} \mathrm{oz}$. daily crystalluria is rarely seen. Eight-hourly oral dosage avoids the disturbance of patient, household or nurse during the night, while unpleasant injections are unneeded. Should the weather be hot and perspiration profuse it may be difficult to maintain an adequate urinary output, when a combination of equal parts sulphadiazine, sulphamerazine and sulphacetamide as advocated by Lehr (1950) is the best method of administering this type of drug. A satisfactory fall of temperature should be obtained within 48 hours. If this does not occur a full reassessment of the whole case should be made. The organisms obtained from the sputum should now be known and their responsiveness to chemotherapy can be assayed. A change in treatment can often be beneficial at this stage. It should be stressed that the effective treatment of pneumonia demands, even more than previously, a constant daily watchfulness of the general condition, physical signs and temperature chart.

Where a satisfactory response has been obtained with sulphonamides the drug can be discontinued three days after the temperature returns to normal and should rarely, if ever, be given for more than six days. With this regime drug fever, leucopenia or drug rashes are rarely met unless there has been previous sensitization.

Should the patient be old, dehydrated, very ill, suffering from nephritis or congestive heart failure, sulphonamides are contraindicated. Here penicillin is the drug of choice and the minimal dose should be 250,000 units of soluble penicillin four-hourly intramuscularly. A low fluid intake is of some advantage for 48 hours in many of these cases, as a high blood concentration ensures a rapid bactericidal effect. Should facilities for intramuscular injection not be available a trial can be given to either chloramphenicol or aureomycin by mouth. Dosage and toxic effects will be discussed later.
Four-hourly injections should be continued until 24 hours after the temperature has returned to normal, which should occur within 60 hours of the commencement of treatment. The dose can then be changed to 500,000 units 12-hourly for not more than three days. There is little justification for using delayed action penicillin in the treatment of pneumonia, for the initial response is not so good, the local discomfort from injection more marked and the probability of sensitization is higher.

Failure to obtain a satisfactory response in 48 hours from either sulphamerazine or penicillin therapy should result in a complete reassessment of the whole case. Frequent causes of failure are as follows:-

I. Infection by a non-responsive organism.

2. Inadequate blood levels.

3. Synpneumonic effusion.

4. Acute pulmonary tuberculosis with pneumonic onset.

5. Pneumonia supervening upon carcinoma.

6. Septicaemia with metastatic inflammatory lesions.

7. Sensitization to drug used.

Attention to these possibilities will usually suggest a further modification of approach.

Staphylococcal pneumonia is most frequently meto with during an influenzal epidemic when its incidence may be high. It is suggested that becauses of this fact sulphonamide therapy should not be used during such an epidemic, but that as far as present knowledge goes penicillin should be given. Where staphylococcal infection of the lung is proved or suspected, dosage should be high, commencing with at least 300,000 units fourhourly and, if response is slow, increasing to 500,000 units four-hourly, combining this with dihydrostreptomycin sulphate, 1 gm. intramuscularly at 12-hourly intervals. The sensitivity of the staphylococcus to the available antibiotics should be tested at once. Penicillin-resistant strains are said to be extremely common in some series, but we have seen very few examples in this hospital amongst the organisms causing pneumonia. Sensitivity is a relative term and is, of course, directly related to the concentration of penicillin in the tissues affected. It is essential that early effective treatment should be assured otherwise breakdown of lung tissue can take place within five or six days. Treatment also should be much more prolonged and should be continued until the sedimentation rate has returned to $15 \mathrm{~mm}$. in one hour and X-ray changes are minimal.

$B$. Friedlander pneumonia most frequently supervenes upon a previously damaged lung, is a rare infection and carries a poor prognosis. Good response will usually follow treatment by strepto- 
mycin in full doses, combined with penicillin 300,000 units four-hourly. Chloramphenicol should be used if no effective response is obtained in three days.

$H$. influenzae is found much more frequently in the sputum following treatment by antibiotics. It is often difficult to say whether it is pathogenic, but if it occurs as the only organism early in the disease or if a pure growth is obtained later when the patient's response is not good, its pathogenicity should be suspected. Its sensitivity should be tested but most strains will be responsive to streptomycin which should be given both intramuscularly, I gm. 12-hourly, and by inhalation, the dosage being $\mathrm{I} \mathrm{gm}$. in $4 \mathrm{cc}$. sterile water, I cc. nebulized four-hourly.

Mixed infections giving rise to pneumonia occur most frequently during a virus epidemic resulting in lowering of local bronchial and general resistance. Infected atelectasis also will yield sputum containing a mixture of organisms. It is probable that in earlier times a number of these progressed to lung abscesses. Response is usually obtained to either a combination of sulphamerazine and penicillin or by following this with adequate doses of chloramphenicol.

Bacterial pleural effusion and empyema. Treatment of pneumonia should involve the daily examination of the lungs to detect the formation of pleural fluid at the earliest moment. In this diagnosis percussion is the most useful method and an increasingly impaired note becoming almost dull is the important evidence of fluid. It is more reliable and less expensive than radiology. Exploration of the chest should be undertaken whenever signs are suggestive of an effusion. The presence of frank pus in the pleura following pneumonia indicates neglect in the early diagnosis and treatment of an effusion. Thick pus is rarely present in less than ten days from the first appearance of fluid, and consequently time is available in which to prevent its formation. If, on first exploration, clear fluid is obtained this should be aspirated completely and systemic treatment continued. Should the fluid be, or become, turbid, aspiration should proceed as previously, bacteriological examination should be undertaken and 300,000 units of penicillin at body temperature injected into the pleura. This operation should be repeated on alternate days until further fluid ceases to form. Thus empyema will be averted. Should pus inadvertently form, aspiration as advocated by Fatti et al. (1946) should be done and a suitable antibiotic, either penicillin or streptomycin, inserted. Intercostal tubes are rarely warranted now. Should pus have been present for several weeks it is as well for the chest surgeon to be associated with the physician in order that an agreed programme of treatment may be carried out. Davies and Asher (195I) have recently shown how little surgical intervention is needed in dealing with this established condition.

Lung abscess. Early diagnosis of this condition is the greatest safeguard in ensuring successful medical treatment. The diagnosis is dependent on a careful history, assessment of physical signs, $\mathrm{X}$-ray examination and an appreciation that purulent breakdown of lung tissue is not uncommon. It is unusual to find a pure growth of any organism except in staphylococcal cases. The essentials in treatment are to maintain free drainage from the earliest moment and to give adequate systemic and intrabronchial therapy. Once again penicillin is effective in most cases, but it should be given in large doses, 300,000 units four-hourly intramuscularly, while 50,000 units six-hourly should be given through an inhaler, using oxygen to nebulize the solution. The collaboration of chest surgeon and physician is essential, for bronchoscopy is needed in practically all cases, but further surgical procedures can now be dispensed with where diagnosis has been early, treatment energetic and where carcinoma as a predisposing cause can be excluded. Penicillin or occasionally streptomycin should be continued until sputum ceases (if no lung disease was present previously), the sedimentation rate returns to normal and $\mathrm{X}$-rays show no evidence of cavitation. Dosage can be reduced considerably one week after the temperature has returned to normal. All possible sources of infection such as septic teeth or pus-filled sinuses should be investigated and treated before the patient is discharged from hospital, and preferably while the antibiotic is being continued.

Chronic suppurative pneumonia. It is doubtful if this condition will be one of major proportion now that lung infections are treated much earlier and more effectively. It is worthy of note that most reported cases occurred before the present range of antibiotics was fully available. Consequently it is not felt necessary to devote space to a disappearing entity. Should a case be encountered careful and repeated bacteriological and virus assay should be done. Therapy, modified as a result of these findings, must be prolonged and collaboration between physician and chest surgeon must be very close.

(b) Virus pneumonia. The following classification is suggested:-

r. Influenzal pneumonia.

2. Primary atypical pneumonia.

3. Pneumonia due to unknown virus.

4. Psittacosis or ornithosis pneumonia.

5. Known virus infection occasionally causing pneumonia, i.e. measles, smallpox, etc. 
6. Rickettsial pneumonia, including $Q$ fever.

Influenzal pneumonia. No known antibiotics have been shown to affect the influenzal virus, but as has been stated staphylococcal infection frequently supervenes. Fulminating cases are due to a rapid spread of this organism throughout the whole bronchial and lung field and its equally rapid and dramatic entry into the blood stream. Dramatic measures only can be advocated in such cases and we have no sure data on which to dogmatize. Intravenous penicillin, $1,000,000$ units, associated with intramuscular penicillin, 500,000 units, two-hourly, and streptomycin, 2 gm. six-hourly, can be combined with intravenous fluids, oxygen and other supportive measures. A trial has been given to intravenous aureomycin using sodium glycinate compound. Results are inconclusive.

Primary atypical pneumonia. Diagnosis of this condition is difficult where no clear-cut epidemic exists. This is unfortunate for the best therapeutic results seem to be obtained if treatment can be given early in the disease. Aureomycin orally is probably most effective and should be given in doses of I gm. initially, followed by $0.5 \mathrm{gm}$. sixhourly. A fall of temperature in 48 hours should follow if the diagnosis is correct and early treatment instituted. The minimal effective doses of aureomycin and chloramphenicol have not yet been agreed. For mild infections that suggested above is adequate, but for those more severely ill twice this initial and subsequent amount should be used. Dosage can be halved three days after the temperature is normal and discontinued on the fifth or sixth day. Little benefit can be expected from continuing treatment longer than ten days, and toxic effects will be probable at this stage. It is found that these are much more common in patients with a history of allergic reactions. Both drugs tend to cause some depression and a feeling of general ill health. Ten to 15 per cent. of patients will experience soreness of the tongue and angles of the mouth resulting from a glossitis and angular stomatitis. In a proportion of these the symptoms will be succeeded by nausea, occasional vomiting, loss of appetite and diarrhoea. Vaginal irritation and soreness is common at this stage.

It is assumed that these effects result from a vitamin $\mathrm{B}$ deficiency conditioned by the alteration of intestinal flora as a result of the bactericidal effect of the drug. Vitamin B should be given from the outset in those with an allergic history, but this does not always prevent the development of these unfortunate effects. Erythemato-urticarial rashes associated with a rise in temperature may also be seen and 'drug fever' must be borne in mind when an unexplained rise of temperature takes place. Too often such a rise is the signal to the inexperienced to add yet one more antibiotic to the unfortunate patient's burden. Courage to stop all treatment is necessary in these days when the giving of yet another new drug is often felt to be the test of a physician's efficiency.

Psittacosis or ornithosis pneumonia. Although some good results have been reported in this condition from the use of penicillin in large doses, it seems probable that aureomycin or chloramphenicol in full doses are likely to give more satisfactory responses. They should certainly be given as soon as the diagnosis is suspected for, as in many virus infections, delay in initial treatment often results in partial response and a delay in complete resolution. In this case fibrosis of the lung is not an uncommon sequela to prolonged infection. It is difficult to be dogmatic as to the results of therapy in a condition so rare as this, in which controlled observation is difficult.

$Q$. fever. The possible clinical importance of this infection has been stressed increasingly both in this country and in America in recent years. Fortunately reported cases are still rare here. The drugs of choice are either aureomycin or chloramphenicol. Here, too, a controlled series is not available on which reliable figures for the therapeutic efficiency can be based.

Acute bronchitis. This condition does not calf̊ for chemotherapy in the majority of cases for res in bed and symptomatic treatment will result in satisfactory resolution in a few days. However there are many sufferers from chronic chest disease in whom acute bronchitis often progresses to suppurative bronchitis and bronchiolitis, and this may lead to a fatal termination. It is true that this combination of conditions is probably leading to more deaths in this country now than uncomplicated pneumonia. The extent of it has been unmasked by the favourable response of the latter to treatment. Consequently a more detailed review of the condition is called for.

Acute bronchitis and bronchiolitis complicating chronic pulmonary disease. This is seen most frequently during the winter months, usually in association with mild but widespread epidemics of upper respiratory infections, virus in origin, combined with the normal bacterial superinfection. The patients are most frequently sufferers from chronic bronchitis, often with increasing bronchitic spasm, emphysema, fibrosis of the lungs or bronchiectasis. A combination of all these conditions is not infrequent while silicosis or other industrial fibrosis may be the basis of the symptoms. The diminished respiratory capacity associated with bronchial narrowing will result in the acute infection giving rise to a respiratory emergency and not infrequently to death from bronchiolar obstruction. Pneumonic consolida- 
tion is rare, but areas of collapse are common and add to the patient's distress.

Clinical course. The condition is seen most frequently in men beyond middle age, the sex difference being determined in part by occupational hazards and excessive smoking over many years. However familial predisposition is an important factor. The onset is typified by the usual coryza and upper respiratory symptoms, but cough soon becomes extremely troublesome. This is associated with substernal pain and soreness, sputum at this stage being scanty and viscid, while the temperature may not be raised. As the infection travels rapidly to the smaller bronchi the general condition deteriorates. Cyanosis and dyspnoea increase, whilst tachycardia, with a falling blood pressure, is marked. The patient often becomes restless, tightness of the chest associated with a fear of suffocation prevents sleep, while if effective doses of sedatives are given fatal suffocation will result. The patient becomes critically ill and anoxaemia with delirium and disorientation, especially at night, so exhaust him that the cough weakens and mucopurulent secretion collects increasingly in the bronchi. Unconsciousness precedes death by hours or days in such cases.

Treatment. Difficulty in advising antibiotics arises in part from the fact that these patients are often allergic to many substances and also that the bacterial element in the infection may be unresponsive to penicillin and other antibiotics. However, systemic and inhalation therapy should be instituted early in the condition, and careful assay of the sputum should be carried out. The essentials of treatment may be summarized as:-

I. Early and continued oxygen therapy, ensuring almost roo per cent. concentration in the severe cases, with an oxygen tent.

2. Aspiration of secretion from the trachea and larger bronchi.

3. Drugs to relieve bronchial spasm and to diminish excessive vasodilatation of the bronchial mucosa.

4. A combination of intramuscular penicillin, 250,000 units four-hourly, and streptomycin, I gm. b.d.

5. Inhalation of penicillin, 50,000 units per cc., I cc. four-hourly, given by means of an inhaler using oxygen to vaporize.

6. Suitable sedatives to ensure sleep but not to depress the respiratory centre. Morphia must be used with extreme caution.

7. Maintenance of an effective fluid balance and nutrition. This may necessitate intravenous therapy:

The therapeutic problem presented by this condition is one of the most difficult remaining to the chest physician. It is worthy of much more attention than it has received in this country to date.

Bronchiectasis. Attempts at permanent reduction of sputum by the use of bactericidal action of antibiotics have not been successful in this condition. The constantly changing bacterial flora probably explains this. However, acute episodes of lung infection, so commonly met with, respond dramatically to specific therapy. Pneumococci and streptococci are the commonest pathogens in these conditions and, consequently, sulphamerazine should be given at the outset while full bacteriological investigation is being done.

Present surveys of large series of cases suggest that the use of antibiotics in rèlapse, associated with routine postural drainage, are producing results comparable to those obtained by drastic surgery.

Chest infection and cardiac failure. Despite the emphasis that Sir Thomas Lewis and others laid on the importance of infection in heart failure, this factor is often overlooked. Cor pulmonale resulting from long-standing disease of the lungs is well recognized, and the deterioration which follows each major chest infection needs no stressing. The more important conditions are those in which hypertensive left ventricular failure is associated with a generalized bronchiolitis, or when a heart previously damaged by coronary occlusion fails because of associated lung infection. Many patients, too, with chronic rheumatic carditis lapse into congestive failure for the same reason. A careful history of the symptoms immediately preceding the breakdown is of importance. The presence of even slight temperature must be treated seriously, for the physical signs in the lungs may be quite equivocal. In left ventricular failure with pulmonary oedema and subacute bronchiolitis, the occurrence of sibilant rhonchi and generalized fine râles may be explained on either a cardiac or pulmonary basis. The use of 'Estopen' penicillin may make inhalation therapy redundant, for it is said to be excreted into the sputum in high concentrations. Further trials of this are proceeding but experience so far is encouraging. However, a raised sedimentation rate will often be decisive, for this is not found in cardiac failure unassociated with infection.

Radiology is not often helpful for much of the infection is intrabronchial, and where there is consolidation it is almost impossible to distinguish this from oedema which is always present in these conditions. Fortunately, too, in intravenous aminophylline we have a drug which will give relief from the extreme dyspnoea resulting from a combination of the failing left ventricle and bronchial spasm, which so often supervenes where 
infection is the precipitating factor. Intramuscular penicillin, combined where indicated, with inhalation therapy should be commenced at once if chest infection is felt to be a contributory factor in cardiac breakdown. Meanwhile investigations should be started and modification of treatment may be made as indicated. The effective treatment of the lung lesion associated with the correct approach to the heart condition may lead to dramatic improvement in what may otherwise seem to be a hopeless problem.

\section{Conclusions}

I. Dogmatism must be avoided when con- sidering the use of antibiotics in non-tuberculous $\cong$ disease of the chest.

2. Bacteriological investigation and sensitivity $\stackrel{\circledR}{\complement}$ tests of organisms obtained are essential.

3. Blunderbuss therapy must not be used.

4. Newly discovered and comparatively untested antibiotics must not easily replace tried remedies.

5. The wider aspects of aetiology such as social factors must be evaluated.

6. The important part that lung infection plays in heart failure has been touched upon.

I should like to thank my colleagues, and many $\vec{\circ}$ others in the hospital for their constant cooperation and help with these problems.

\title{
BIBLIOGRAPHY
}

GUNNISON, J. B., COLEMAN, V. R., and JAWITZ, E. (I950a), Proc. Soc. exp. Biol. N.Y., 75, 549; (1950b), f. lab. Clin. Med., 36, 900 .

LEHR, D. (1950b), Brit. med. F., ii, 601.
FATTI, L., FLOREY, M. E., JOULES, H., HUMPHREY, J. H., and SAKULA, J. (1946), Lancet, i, 295.

DAVIES, D., and ASHER, R. A. J. (1951), Lancet, i, 924.

\section{THE CLINICAL ASSESSMENT OF DISPROPORTION *}

\author{
By William Hunter, M.D., F.R.C.O.G. \\ Obstetrician, Princess Mary Maternity Hospital; Associate Surgeon, Royal Victoria Infirmary, Newcastle upon \\ Tyne; Senior Obstetrician and Gynaecologist, North-West Durham Hospital Group
}

Attention has been devoted during recent years to radiological methods of assessment of the sizes of the maternal pelvis and foetal head and much has been achieved. The greatest strides have been made in the estimation of the size of the pelvis, but progress has not been limited to actual measurement of the bony structures. There is now a better understanding of the architecture of the pelvis - of its variations in shape as well as in size - and of the significance of these variations. So much thought has, in fact, been directed into these comparatively new channels that many of the methods of clinical assessment previously in use seem to have been forgotten or to have fallen largely into disuse. For this reason the time now seems opportune to re-consider some of the clinical methods available for estimating the relative sizes of the foetal head and the maternal pelvis. It is, after all, upon these methods that we must rely to select those cases which require fuller investigation and ultimately, in conjunction with

*A paper read at a meeting of the Newcastle and Northern Counties Medical Society in Newcastle upon Tyne on Thursday, February I, 1951 . other findings, to decide upon the further manage- $\stackrel{\circ}{\stackrel{\circ}{2}}$ ment of the case. The words of the pious I 8 th $\overrightarrow{0}$ century German midwife, Justine Siegemundin- 3 'All I do depends on God's help and on the skilful motions of my hands'-have a particular? application to this type of case.

Some indication of possible difficulty at the time of the confinement may be discovered during the course of the routine antenatal investigation of a case. The patient may be of small stature, she may have an obvious limp, occasionally due to ankylosis of the hip, or she may show gross de- 음 formities of the long bones or spine and, for in- $\rightarrow$ stance, there may be obvious rachitic manifestations. In giving the medical or surgical history $N$ she may bring forward an account of fractures, tumours or diseases of the pelvic bones or spine, or tumours of the pelvic organs. The previous $\omega$ obstetric history may suggest the possibility of recurrent complications arising in the approaching ${ }^{\circ}$ confinement. There may have been a prolonged $\mathbb{\Phi}$ or complicated labour, a difficult delivery or ${ }^{+}$ Caesarean section, maternal trauma or a stillborn $\frac{T}{0}$ or injured child, possibly of large size, on a pre- $\frac{\mathrm{O}}{\mathbb{D}}$ vious occasion. In all such cases every effort 